Check for updates

Cite this: J. Mater. Chem. A, 2019, 7, 15309

Received 14th April 2019 Accepted 29th May 2019

DOI: $10.1039 / c 9 t a 03950 c$

rsc.li/materials-a

\section{Expanding the upper limits of robustness of cellulose nanocrystal aerogels: outstanding mechanical performance and associated pore compression response of chiral-nematic architectures $\dagger$}

\author{
Anurodh Tripathi, (D) $\ddagger^{\mathrm{ab}}$ Blaise L. Tardy, (D) $\xi^{* \mathrm{~b}}$ Saad A. Khan, ${ }^{a}$ Falk Liebner (D) ${ }^{\mathrm{c}}$ \\ and Orlando J. Rojas (D) *ab
}

Control over the nanoscale architecture of a material enables fine tuning of its physical characteristics and associated functions. Depending on the performance demands, properties such as active surface area, density, optical response, transport characteristics and mechanical resilience can be tailored by nanostructuring. Herein, we exploit the liquid crystalline phase transitions in aqueous dispersions of highly anisometric, nanoscaled and high strength ( $E_{\mathrm{A}}>150 \mathrm{GPa}$ ) cellulose nanocrystals (CNCs) to afford chiral-nematic ordered aerogels with controlled meso- and microstructures. Unprecedented levels of specific strength and toughness were achieved by controlling CNC assembly and derived architectures. We determined that the specific strength, and toughness, of CNC aerogels are improved by up to $137 \%$ and $60 \%$, respectively, compared with the highest reported values for aerogels formed solely from cellulose nanofibrils or nanocrystals. Our results demonstrate that chiral-nematic ordered aerogels with controlled meso- and microstructures replicate the liquid crystalline phase transitions of CNCs in aqueous dispersions. The obtained architectures are evaluated systematically by varying the long-range order of the aqueous CNC dispersion from mostly isotropic to completely anisotropic. The resulting aerogels display a strong relationship between the mesopore fraction and selective light reflection (iridescence) as a function of mechanical load. Specifically, we find that the mechanical performance associated with pore compression under load is greatly enhanced by chiral-nematic ordering. The new limits in the mechanical properties of CNC-based aerogels point to new structural considerations for the synthesis of next generation porous constructs that exploit the inherent long-range order of such building blocks.

\section{Introduction}

Controlling pore sizes, their distribution and their ordering in a given material offers control over its mechanical characteristics; i.e. from extremely soft and tough to strong and brittle. ${ }^{1-3}$ Generally, such porous assemblies include foams, with a macroscale cellular architecture that provides strength,, ${ }^{\mathbf{4 , 5}}$ or aerogels with micro/meso pores and high accessible specific surface

\footnotetext{
${ }^{a}$ Department of Chemical \& Biomolecular Engineering, NC State University, Raleigh, NC 27695-7905, USA

${ }^{b}$ Department of Bioproducts and Biosystems, School of Chemical Engineering, Aalto University, P.O. Box 16300, FIN-00076 Aalto, Espoo, Finland.E-mail: blaise.tardy@ aalto.fi; orlando.rojas@aalto.fi

'Institute for Chemistry of Renewable Resources, University of Natural Resources and Life Sciences Vienna, Konrad-Lorenz-Straße 24, 3430 Tulln, Austria

$\dagger$ Electronic supplementary information (ESI) available. See DOI: $10.1039 /$ c9ta03950c

$\ddagger$ These authors contributed equally.
}

areas at the cost of a relative increase in brittleness, for instance silica aerogels. ${ }^{6,7}$ In biological tissues, such as wood, multi-scale porous architectures coexist and can be used to produce high performance materials by top-down approaches that maintain the hierarchical framework. ${ }^{8-10}$ The versatility in such materials arises principally from the treatment chosen to modify the composition of the bio-structures and also from the biosynthetic process itself, i.e. via modification during the biological fabrication of the material. Conversely, bottom-up construction, i.e. assembling small building blocks into foams or aerogels offers the advantage that materials can be customized for a specific application. The latter approach potentially allows for a more amenable manufacturing process. However, higher costs caused by elaborate processing steps have to be considered as well, which can include the extraction of the required small building blocks from an appropriate matrix. ${ }^{11-13}$ A great advantage on the other hand is that bottom-up approaches enable the formation of constructs that are not commonly 
accessible in nature, as demonstrated for a multitude of synthetic polymeric materials. ${ }^{14}$

Nanocelluloses have recently been heavily investigated with regard to their use as building blocks for the creation of porous materials. ${ }^{13,15}$ Cellulose nanofibers (CNFs) are high axial aspect nanomaterials featuring widths of 3-20 nm and lengths of the order of several microns. They are highly flexible but strong (tensile strength of the order of several GPa). ${ }^{16-18}$ Removal of disordered cellulose chains from CNF structures, via acid-based hydrolysis, yields cellulose nanocrystals (CNCs), which are highly crystalline and extremely strong $\left(E_{\mathrm{A}}>150 \mathrm{GPa}\right) .{ }^{19,20} \mathrm{CNFs}$ and CNCs have low thermal conductivity, low thermal expansion coefficient, ${ }^{21}$ poor solubility in most common solvents ${ }^{22}$ and very high interparticle affinity upon completion of evaporation-induced self-assembly (EISA). ${ }^{23}$ The combination of their high anisometry and nano-scale size with their mechanical strength and chemical inertness renders CNCs ideal building blocks for the fabrication of next generation lightweight materials, such as foams and aerogels. Hitherto, lightweight materials have mostly been fabricated from sol-gel precursors, such as alkoxysilanes, achieving excellent control over transport properties (heat, mass, and sound). However, the brittleness associated with these materials remains a major limitation for most uses. ${ }^{24} \mathrm{~A}$ particularly appealing aspect of using highly anisotropic cellulosic building blocks is associated with their long-range order in aqueous dispersion or in the solid state. ${ }^{21,25,26}$ Specifically, it has been demonstrated that nematic ordering of CNCs and CNFs can greatly enhance the properties of the formed aerogels, for instance, enabling a high directional toughness while maintaining high optical transparency and thermal insulation. ${ }^{21,27}$ Compared to conventional aerogels with isotropic skeletal structures, the porous assemblies of nematic ordered aerogels can be further enhanced by uniaxial compression, whereby pore size harmonization in expense of macropores and in favor of mesopores occurs. ${ }^{21} \mathrm{CNCs}$ can be assembled with a chiral-nematic $(c n)$ order, where each of the nematic planes is arranged helicoidally across the thickness of the material. Chiral-nematic CNC materials $(c n-\mathrm{CNC})$ can be easily obtained using various bottom-up approaches, generally via EISA. The properties of $c n$-CNC materials can be altered by various processes such as topography control and the use of magnetic fields or by the incorporation of additives. ${ }^{28-31}$ Chiral nematic arrangements of nanoparticles are omnipresent in biological systems simultaneously providing high strength and toughness as well as other properties, such as selective light reflection. ${ }^{32}$ While $c n$ ordering occurs naturally in plants and animals, top-down processing to mimic such architectures is challenging, particularly in scales above a few $\mathrm{mm} \cdot{ }^{33-35}$ Herein, we formed $c n$-CNC aerogels gelled by non-solvent-induced phase separation followed by supercritical drying. ${ }^{36}$ The effect of concentration-dependent phase separation and of the EISA to achieve control over the $c n$ order in the aerogels is investigated. We demonstrate that the density, mechanical properties and mesopore volume can be adjusted by controlling the initial state of assembly and gelation. In that endeavor we demonstrate the benefits of controlling the extent of ordered mesopores to form outstandingly robust aerogels with specific strength, and toughness, outperforming currently reported anisotropically ordered, single component cellulose aerogels from individualized cellulose nanofibrils by 137 and $61 \%$ in their respective specific properties. ${ }^{21,27}$ We also demonstrate the scalability of macropores to pore sizes below $100 \mathrm{~nm}$ as a function of the aerogel architecture, wherein a $\sim 130 \%$ increase in the volume of pores with sizes of $2-100 \mathrm{~nm}$ is achieved by partial compression of the $c n$-CNC aerogels. The work presented here further extends the property spaces for cellulosic aerogels and defines the scaling phenomenon associated with their specific strength, specific toughness and long-range order.

\section{Experimental}

\section{Materials}

Cellulose nanocrystals, CNCs (CAS no. 7789-20-0) were produced by the USDA's Forest Products Laboratory (FPL, Madison, WI) and acquired through The Process Development Center at the University of Maine. In their production, sulfuric acid hydrolysis was carried out using dissolving grade wood fibers suspended in water. The CNCs were $20 \mathrm{~nm}$ in width and $150-300 \mathrm{~nm}$ in length and their sulphur content was $0.95 \mathrm{wt} \%$. Further results from the characterization of the CNCs can be found in our previous study. ${ }^{26}$ Milli-Q water (U.S.A. Millipore, Synergy UV) was used to dilute the CNC dispersion. Acetone was obtained from VWR Chemicals International S.A.S. and liquid $\mathrm{CO}_{2}$ was obtained from AGA Oy, Finland.

\section{Synthesis of CNC aerogels}

An $11.1 \mathrm{wt} \% \mathrm{CNC}$ aqueous dispersion was diluted to concentrations of 4, 5.5 and $7 \mathrm{wt} \%$ by adding an adequate amount of Milli-Q water. $50 \mathrm{~mL}$ of each of these dispersions were left to equilibrate for 30 days in a flat-base cylindrical container equipped with a screw top. Thereafter, $50 \mathrm{~mL}$ of acetone was carefully added on top of the dispersion while minimizing any disturbance of the CNC dispersion with the acetone interface and to initiate non-solvent induced phase separation. Supernatant removal and replacement with fresh acetone were performed multiple times over the course of one week with increasing volumes of acetone until a solid gel was obtained.

In another set of experiments, water was evaporated from the $5 \mathrm{wt} \% \mathrm{CNC}$ dispersion under ambient conditions $\left(21{ }^{\circ} \mathrm{C}\right.$ and $23 \%$ humidity) to achieve final concentrations of 10,20 and $25 \mathrm{wt} \%$, where the dispersions were gelled. This was carried out to evaluate the effect of evaporation-induced self-assembly (EISA) on the final arrangement of CNCs in the aerogels, their pore structure and mechanical properties. These EISA samples were also subjected to acetone exchange as described above. The samples obtained after solvent exchange are referred to as "organogels".

The gels were then isolated and cut into small cuboids ( $c a .2$ $\mathrm{cm}^{3}$ each). Critical point drying was performed with a Leica EM CPD300. Twenty-five cycles of liquid $\mathrm{CO}_{2}$ exchange $\left(10{ }^{\circ} \mathrm{C}, 3\right.$ samples per batch) were conducted to completely replace acetone with liquid $\mathrm{CO}_{2}$. Thereafter, the chamber was heated to $40^{\circ} \mathrm{C}$ (for $1 \mathrm{~h}$ to reach $75 \mathrm{bar}$ ) and the $\mathrm{CO}_{2}$ was vented off slowly. 
All CNC aerogels were stored in an airtight container at ambient temperature until further use. The aerogels obtained by nonsolvent induced gelation from dilute CNC dispersions are referred to herein as AG-4, AG-5.5, and AG-7 reflecting their respective initial solid contents. The aerogels obtained by EISA and subsequent non-solvent induced gelation were labelled according to the CNC content of the dispersions after EISA, namely, EISA-10, EISA-20, and EISA-25.

\section{Gel and aerogel characterization}

The anisotropic volume fraction of the equilibrated CNC dispersion was recorded by normalizing the height at the phase boundary between the isotropic and anisotropic phases with respect to the initial dispersion height, as reported before. ${ }^{26}$ After the equilibrated dispersion was gelled using acetone, the gel fraction was recorded as the final height of the phaseseparated gel, normalized with the initial CNC dispersion height. The reduction in CNC dispersion volume was reported as gel volume shrinkage, which consequently resulted in the increase in CNC concentration, reported as final concentration after gelation.

The volume of CNC aerogels was calculated from their spatial dimensions and the CNC aerogel bulk density was reported as mass per unit volume. The volume shrinkage of the gels (VS_Gel) was calculated as

$$
\text { VS_Gel }=\frac{V_{\text {dispersion }}-V_{\text {gel }}}{V_{\text {dispersion }}}
$$

whereas volumetric shrinkage of the aerogels (VS_Aerogel) during critical point drying was calculated as

$$
\text { VS_Aerogel }=\frac{V_{\text {gel }}-V_{\text {aerogel }}}{V_{\text {gel }}}
$$

The porosity of the aerogel was calculated from the bulk density by assuming the density of single CNC to be $1.6 \mathrm{~g} \mathrm{~cm}^{-3}$, using the following equation:

$$
\text { Porosity }(\%)=1-\frac{\text { bulk density }_{\text {aerogel }}}{1.6} \times 100
$$

The CNC aerogels were fractured, and their cross-sections were imaged with a Sigma Zeiss ULTRA-plus Scanning Electron Microscope (SEM). Sample preparation was accomplished by puncturing the surface of the aerogels with a pair of sharp tweezers. The applied force fractured the aerogel with propagation of the fracture along the $c n$ director along the thickness of the material, as commonly observed for such architectures (e.g. as described in ref. 8). This freshly cleaved surface was sputter coated and exposed to the electron beam after adhering the sample to an SEM stub using carbon tape. Importantly, the direction of the fracture in the $Z$ direction was highly dependent on the homogeneity of the long-range order and Bouligand cuts may occur for non-orthogonal fracture propagation (i.e. fractures that are not directly orthogonal to the $c n$ director). This results in pitches appearing larger than their actual size. This is clearly illustrated, for instance, in the top section of
Fig. S1(b3). $\uparrow$ The domains of the aerogels imaged were chosen based on smaller observed pitches that reflected fracture propagation following the cholesteric director more closely.

Mechanical testing was performed using an INSTRON 4204. The cuboidal samples of known dimensions with a height of $1 \mathrm{~cm}$ were compressed using a load of $1 \mathrm{kN}$ that was lowered at the rate of $1 \mathrm{~mm} \mathrm{~min}^{-1}$. The aerogels were compressed in the direction parallel to the $c n$ director. The compression modulus or stiffness of the given aerogel was reported as the slope of the respective stress-strain curve at $0.5 \%$ strain. The toughness of the aerogel was calculated from the area under the stress-strain curve up to $70 \%$ strain. The strength of the aerogel was recorded as the stress measured at $70 \%$ strain. All these values were normalized with the bulk density of the aerogel to yield the specific modulus, specific toughness and specific strength, respectively.

Nitrogen adsorption and desorption isotherms at $-196{ }^{\circ} \mathrm{C}$ were recorded using a Micromeritics ASAP 2020 (TriStar II). Prior to measurement, the samples were degassed for 15 hours at $97{ }^{\circ} \mathrm{C}$ under a $\mathrm{N}_{2}$ atmosphere. The Brunauer-Emmett-Teller (BET) surface area was calculated from the adsorption branch of the isotherm using data points of the relative pressure range of 0.1-0.3. Pore size distribution and pore volume were calculated from the desorption branch using the Barrett-Joyner-Halenda (BJH) method.

\section{Results and discussion}

\section{Aerogel synthesis and structure}

The concentration-dependent phase separation and long-range ordering of CNCs in dispersion as employed herein for the preparation of anisotropic gels, and aerogels, are shown schematically in Fig. 1. The preparation of $c n$-CNC aerogels was conducted via (1) non-solvent (acetone) induced gelation of dispersions and (2) evaporation induced self-assembly (EISA) of CNCs at different solid contents (Fig. 1a and b). For non-solvent induced gelation, CNC dispersions (4, 5.5 and $7 \mathrm{wt} \%)$ were left undisturbed until equilibrium was reached, yielding an upper, isotropic and a bottom, anisotropic phase. ${ }^{26}$ While the isotropic phases are dominated by randomly oriented CNCs, $c n$ tactoids coexist with fused domains in the anisotropic volume fractions. The latter have higher specific density compared to the isotropic top layers. The volume fractions of the respective phases whose ratios change with variation of the total CNC content are listed in Table S1. $\dagger$ In another set of experiments expanding the investigation of evaporation induced self-assembly (EISA), a $5 \mathrm{wt} \%$ dispersion of CNCs was concentrated to different levels of solid content $(10,20$ and $25 \mathrm{wt} \%)$ by slow evaporation of water. The hierarchically structured architecture was uniaxially densified by evaporation, yielding large nematic "planes" arranged in a chiral nematic, helicoidal order across the thickness of the assembly, as previously reported. ${ }^{26,37,38}$ This results from the kinetic arrest concentration, which forces respective dispersions to gel at solid contents beyond $7 \mathrm{wt} \%{ }^{25}$

Both the equilibrium phase-separated samples consisting of upper isotropic and lower anisotropic phases as well as the EISA samples were subjected to solvent exchange from water to 
a)

CNC Suspensions

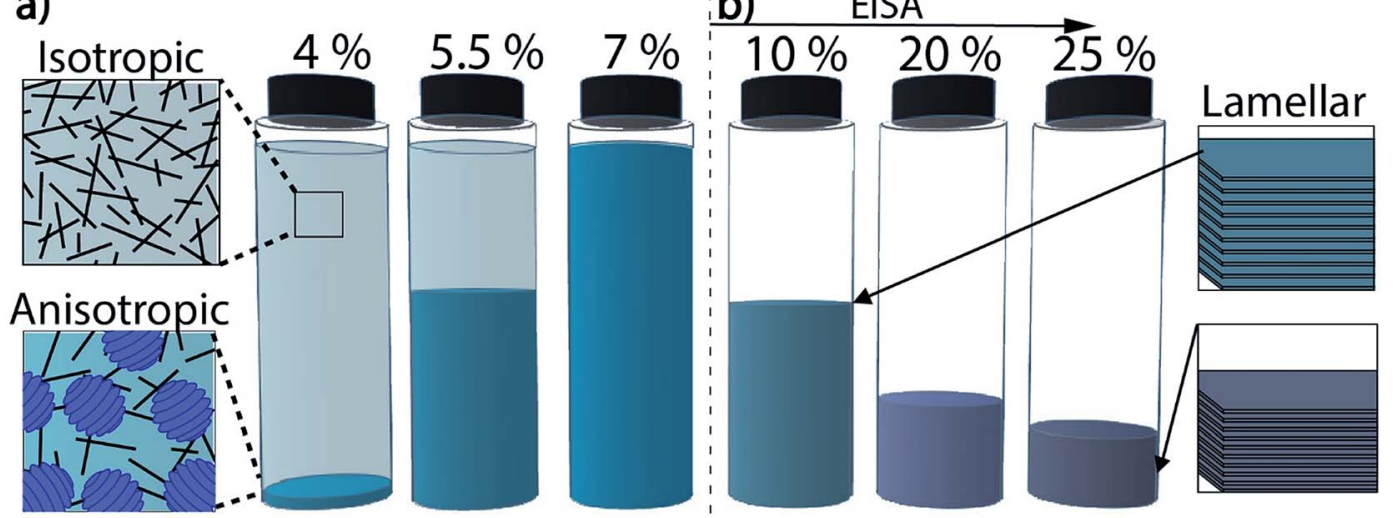

Gelation by solvent-exchange Supercritical drying
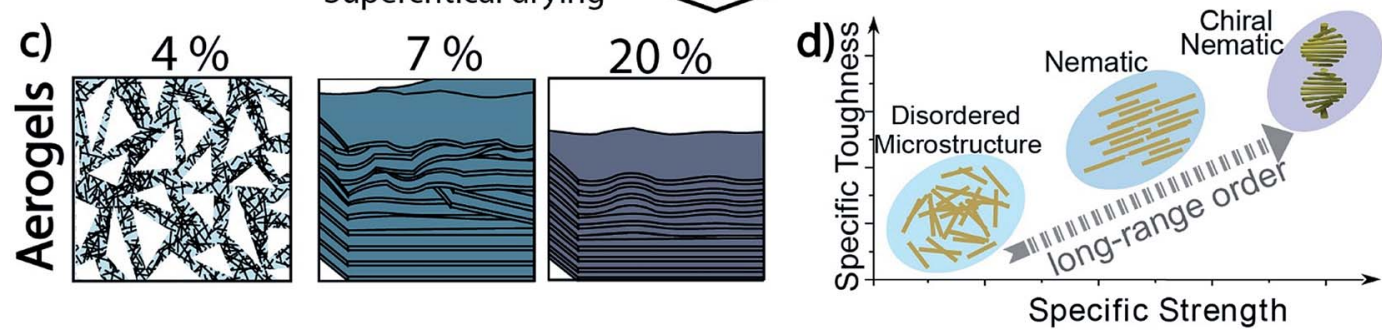

Fig. 1 Schematic representation of the two types of precursor dispersions used for CNC aerogel preparation, (a) equilibrated CNC dispersions of varying concentrations (4,5.5, and $7 \mathrm{wt} \%$ ) and (b) CNC dispersions obtained by evaporation induced self-assembly (EISA) with final CNC concentrations of 10, 20 and 25 wt\% exhibiting increasing long-range order. "Lamellar" herein refers to annealed cn domains. (c) Schematic representation of corresponding aerogels with a highly disordered, isotropic fraction shown for the $4 \%$ aerogel, whereas a long-range order is shown for $7 \%$ and $20 \%$ aerogels. (d) A representation of the order-dependent properties described throughout the study (further described in Fig. 4).

acetone by multiple solvent exchanges steps (see Experimental section for the solvent exchange procedure). A high volume shrinkage was observed for the non-solvent induced gelation of the equilibrated CNC dispersions, i.e. non EISA samples. The gels formed from these dispersions featured a highly percolated CNC network morphology. Compared to the volume of the initial dispersions, reductions in volume of 41,59 and $59 \%$ were observed for the dispersions containing 4, 5.5 and $7 \mathrm{wt} \% \mathrm{CNC}$, respectively (Table S1 $\dagger$ ). The resulting high CNC content afforded dense transparent gels that underwent little volume reduction (1.9-6.1\%), during the final supercritical carbon dioxide drying, yielding aerogels. The obtained aerogels were named AG-4, 5.5 and 7 corresponding to the respective CNC contents of the initial dispersions. It is worth mentioning that the final CNC content (wt\%) of the gels increased from the initial CNC content (wt\%) as follows: $12.4(4$ initial $)<14.8(5.5)<$ $17.2(7)$.

CNC dispersions of higher solid content ( $\geq 10 \mathrm{wt} \%$ ) as obtained by EISA of a $5 \mathrm{wt} \%$ CNC dispersion exhibited negligible volume contraction upon infusion of the non-solvent, acetone, that triggered the formation of semi-transparent gels. The obtained aerogels were named EISA-10, 20 and 25 corresponding to the respective final $\mathrm{CNC}$ concentration.

All solvent exchanged gels were converted to aerogels via supercritical carbon dioxide drying (details in the Experimental section). Comprehensive morphological, mechanical and pore analyses, as detailed below, confirmed that the properties of the aerogels are closely correlated with the CNC content of the dispersions prior to non-solvent induced gel formation, which defined the respective order from isotropic to $c n$ (Fig. 1c and d).

Backlit images of organogels, in acetone, formed after solvent exchange are shown in Fig. 2a. The arrows in the figure indicate the observed phase boundary between the isotropic and anisotropic phases in gels formed from CNC dispersions at concentrations of 4 and $5.5 \mathrm{wt} \%$. Compared to the volume shrinkage that occurred during solvent exchange (40-60\%), the formation of aerogel by supercritical drying caused only a minimal further densification (volume reduction of $<7 \%$, Fig. 2b). Therefore, the density of the aerogels correlated well with the CNC concentration in the gels, which was also intimately linked with the initial aqueous dispersion concentration as shown by the black line plotted in Fig. $2 b$.

Fractured surfaces of the aerogels obtained from different precursor CNC concentrations were observed by SEM to identify the macroscopic arrangements and long-range orientation of the nanoparticles. All fractures ( $c f$. Experimental section) were observed to be perpendicular to the main plane of the formed aerogels, i.e. across the principal plane of orientation of CNCs. SEM images of the top and bottom cross-section of CNC aerogels are presented in Fig. 3 and S1. $\dagger$ The $4 \mathrm{wt} \%$ CNC dispersion 
a)

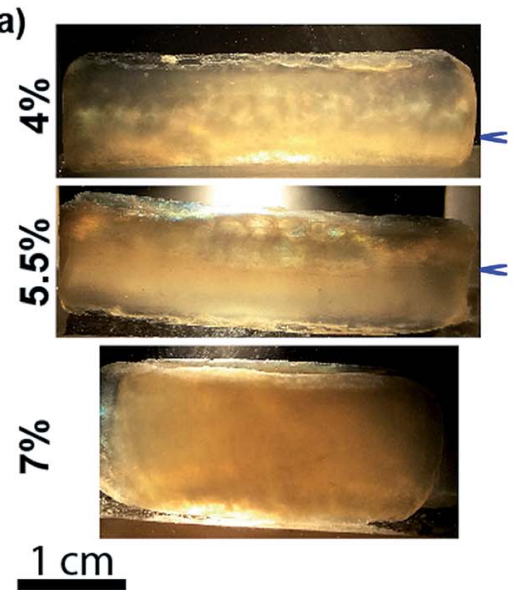

b)

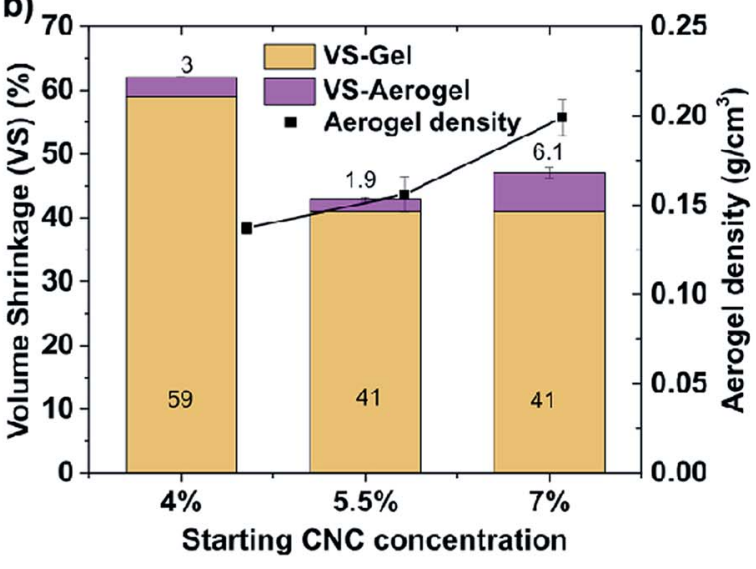

Fig. 2 (a) Backlit gels formed after solvent exchange in acetone. The labels on the left-side indicate the concentrations of the precursor dispersions. Blue arrows indicate transitions from the chiral nematic fraction (bottom) to the isotropic one (top). (b) Volume shrinkage associated with gel formation (yellow) and aerogel preparation (purple). The corresponding values are shown in the bar graph. The total shrinkage is the sum of the two values. Aerogel density is also reported in the graph.

displayed an upper, isotropic domain and a bottom, anisotropic domain. Consequently, as displayed in Fig. 3(a1), a disordered pore structure is observed in the top cross-section of the AG-4 aerogel, whereas the bottom cross-section of the same system shows preserved nematic planes with large interlayer distances (Fig. 3(b1)). A zoomed-out image of the AG-4 bottom crosssection shows a corrugated/wrinkled structure perpendicular to the $c n$ director, as shown in Fig. S2. $\dagger$ The latter effect can be ascribed to the direction-dependent solvent-intrusion, which may have led to preferential aggregation in one direction within the nematic planes. A cross section of AG-5.5 displayed in Fig. S3† clearly shows the transition between anisotropic and isotropic phases with a seemingly increased corrugation in the anisotropic domain. A similar corrugated structure is also observed in the upper cross-section of the AG-7 aerogel
(Fig. 3(a2)), with the respective precursor aqueous dispersion forming a principally anisotropic phase. The fingerprint of ordered $c n$ structures is observed both atop (Fig. 3(a2)) and at the bottom (Fig. 3(b2)) of the cross-sections of AG-7 aerogel.

EISA from $5 \mathrm{wt} \%$ CNC dispersions to $20 \mathrm{wt} \%$ dispersions (namely, EISA-20) resulted in the development of long-ranged $c n$ domains, as seen before. ${ }^{26}$ The hierarchical order is evident in the SEM images of corresponding EISA-20 aerogel samples. A reduction in pitch with increasing CNC concentration can be seen from the SEM image of the bottom cross-section of AG-4 and AG-7 as well as EISA-20 shown in Fig. 3b, with specific reflections (structural colors) observed for the EISA-20 gels (Fig. S4†). The top cross-section of the EISA-20 aerogel also exhibits a similar corrugation of the nematic domains (Fig. 3(a3)) and well-oriented $c n$ structures in the bottom
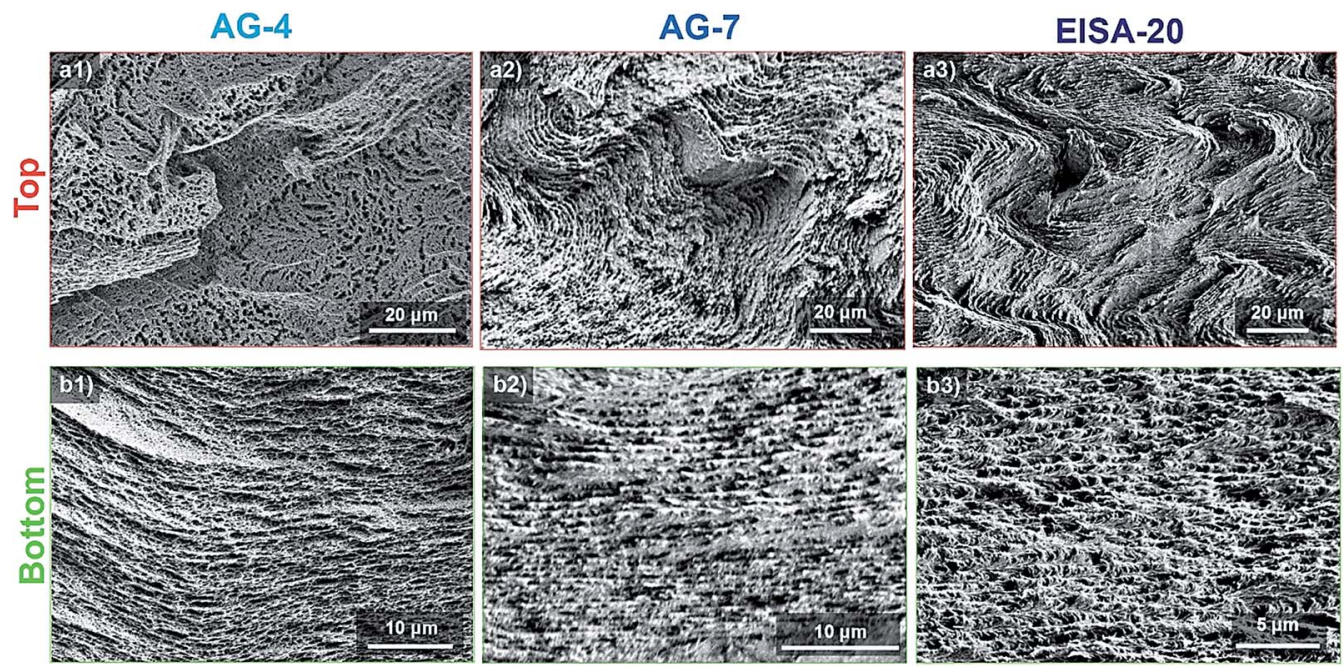

Fig. 3 SEM images of the aerogel cross-sections observed perpendicular to the principal plane of the assembly, across $\mathrm{cn}$ pitches. The top row exhibits images near the top cross-section, (a1) AG-4, (a2) AG-7, and (a3) EISA-20 (scale bars: $20 \mu \mathrm{m}$ ). The bottom row exhibits images near the bottom cross section, (b1) AG-4, (b2) AG-7 (scale bars: $10 \mu \mathrm{m}$ ), and (b3) EISA-20 (scale bar: $5 \mu \mathrm{m}$ ). 
(Fig. 3(b3)). This highlights the effect of the rate of acetone diffusion during solvent-assisted gelation. The faster intrusion led to more significant deformation in the upper layers, in contrast to the bottom layers. The fact that such deformations are present across the AG-4 sample (Fig. S2 $\dagger$ ) suggests relatively unhindered diffusion of acetone coupled to limited structural integrity of the low concentration dispersion, which particularly matters in the initial stages of gelation.

From the data presented so far, it can be observed that the $\mathrm{cn}$ structure of CNCs is well-preserved within the aerogels and that its fused domains correspond well with the dispersion behavior suggested in Fig. 1 and 2a. However, the solvent exchange may significantly affect the macro-scale orientation of $c n$ domains generating hierarchical structures with wavy corrugated macrodomains.

\section{Aerogel long range order and mechanical attributes}

The existence of a long-range $c n$ order is often associated with mechanical toughness and strength, as is the case in most natural structures possessing such an order, e.g., as observed in the armor of crustaceans. ${ }^{33,35}$ Herein, up to EISA-10, the aerogels exhibited a density $<0.2 \mathrm{~g} \mathrm{~cm}^{-3}$ (Table S2 and Fig. S5a $\dagger$ ), yet they displayed unusual strength as demonstrated in Fig. S5b, $\uparrow$ which illustrates that a $2 \mathrm{~kg}$ load applied on the aerogel produced no macroscopically visible damage (see inset). This qualitative observation motivated the quantification and comparison of the mechanical properties of CNC aerogels as described in Fig. 1 with other aerogels reported in the literature, as illustrated in Fig. $4 \mathrm{~b}$.

All aerogels were compressed with a maximum load of $1000 \mathrm{~N}$ on a comparatively similar area of applied load, with strains applied in the direction perpendicular to the lamellae, where lamellae refer to well annealed $c n$ domains as depicted in Fig. 1b. The stress-strain profiles are described in Fig. 4a and a summary of the mechanical properties obtained is available in Table S2. $\uparrow$ The stress-strain curves exhibit a small linear elastic region below $1 \%$ strain followed by a plateau and a subsequent densification regime typical of porous architectures (Fig. 4a inset). The obtained Young's modulus of AG-7 (1.90 MPa) compared to that of AG-4 (0.17 MPa) and AG-5.5 (0.45 MPa) indicates a well-developed, compact microstructure that resists compression. In contrast, a high fraction of the disordered, porous structure of AG-4 is compressible (highest compression strain of $\sim 90 \%$ at a stress of 3.1 MPa). The stiffness, toughness, and strength of the aerogels increase (Table S2 $\dagger$ ) as the orientation of CNCs shifts from the disordered AG-4 to the highly ordered cholesteric architecture of AG-7. The EISA-25 aerogel consists of the most ordered and the densest structures as reflected by the highest modulus $(2.50 \mathrm{MPa})$ and the lowest compression strain $(52 \%$ at a stress of 3.0 MPa). The extent of ordering, density and mechanical properties of EISA-10 and 20 are in between those of EISA-25 and AG-7 (Fig. S6 and Table $\mathrm{S} 2 \dagger)$. The increase in long range order is also observed from the values of densification strain as shown in Table S2. $\dagger$ The densification strain is defined as the threshold strain from which stress increases exponentially. This corresponds to the stage where the individual rigid domains collide with each other and is reported as the intersection of tangents drawn at the plateau and densification regions of the stress-strain curves. The densification strain decreases sharply from AG-4 (80\%) to AG-7 (64\%) owing to the well-developed long-range cholesteric order of the latter. A further sharp decrease in the densification strain was observed for the EISA aerogel series, dropping from 52\% for EISA-10 to 30\% for the EISA-25 aerogel.

The visual appearance of the AG-5.5 aerogel under compression following the stress-strain profiling is included in Fig. 4a. Interestingly, upon an intermediate compression of $50 \%$, the initially whitish AG-5.5 aerogel turns reddish and, upon a maximum compression of $76 \%$, transitions to greenish. The observed shifting of light reflection to lower wavelengths indicates a reduction of the well conserved $c n$ pitch. Other aerogels also presented similar compression-induced shifting of their specific reflections (Fig. S7†). The most pronounced difference was observed for AG-4 which transitioned from an initially slightly red reflection towards green and blue reflections when undergoing partial compression and up to $90 \%$ compression, respectively (Fig. S7a $\dagger$ ). AG-4 and AG-5 showed a more pronounced lowering in reflection wavelength, suggesting that their high compliance is important for such attributes. The observed compression-dependent iridescence of $\mathrm{cn}$ CNC aerogels should be further explored since it has the potential to be used in a new class of biobased mechanical aerogel sensors, such as to report damage when used to protect goods under compression. Similar observations have also been previously reported in another study using nanocomposites formed from chiral nematic ordered CNCs embedded in aminoformaldehyde resins. ${ }^{39}$

It is noteworthy that the absolute value of stiffness for the EISA-25 aerogel is 100-times higher than the highest reported stiffness for monolithic, isotropically ordered CNC aerogels produced by Abraham et al. ${ }^{\mathbf{4 0}}$ and 3-times higher than that for nematically oriented CNF aerogels reported by Kobayashi et al. ${ }^{27}$ Furthermore, the absolute values of toughness and strength of AG-7 aerogels are approximately 10 and 15 times higher, respectively, compared to the highest toughness for CNF aerogels measured by the same authors ${ }^{27}$ and by Plappert et $a .^{21}$ Note that the strength of an aerogel, its stiffness (Young's modulus), toughness, and compression resistance are strongly correlated with its density. Therefore, the measured properties were normalized with density to obtain specific-strength and specific-toughness. As such, AG-7 exhibited outstanding specific toughness (2.99 $\mathrm{MPa} \mathrm{cm}^{3} \mathrm{~g}^{-1}$ ) and strength (17.59 $\mathrm{MPa} \mathrm{cm}^{3} \mathrm{~g}^{-1}$ ) compared with previously reported mechanically robust nanocellulose aerogels (Fig. 4b). In particular, the EISA-10 aerogel exhibited the highest specific toughness (3.55 $\left.\mathrm{MPa} \mathrm{cm}^{3} \mathrm{~g}^{-1}\right)$ and strength (21.52 $\mathrm{MPa} \mathrm{cm} \mathrm{c}^{3}$ ) reported so far for monolithic nanocellulose aerogels (Fig. S8†), considerably higher than those for any reported aerogels made from single component CNCs and oriented CNFs. The excellent mechanical properties of AG-7 and EISA-10 aerogels are attributed to a long-range cholesteric order achieved at a comparatively low density. It is noteworthy that the EISA-20 aerogel, with a higher and more packed long-range order, exhibited a decrease in specific 
a)

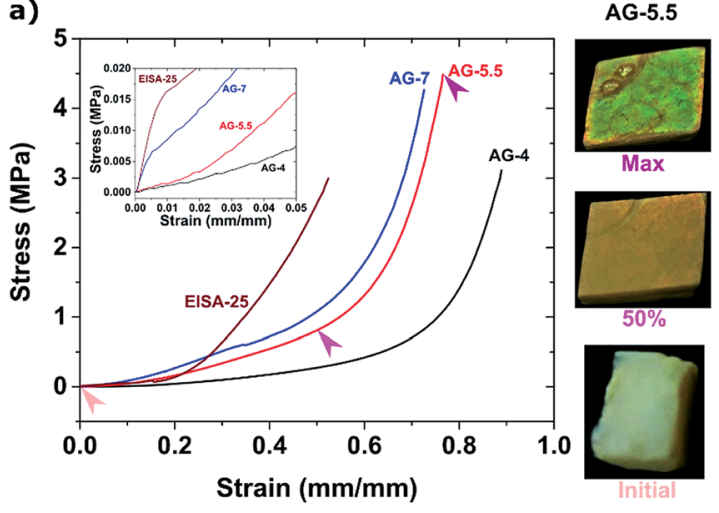

b)

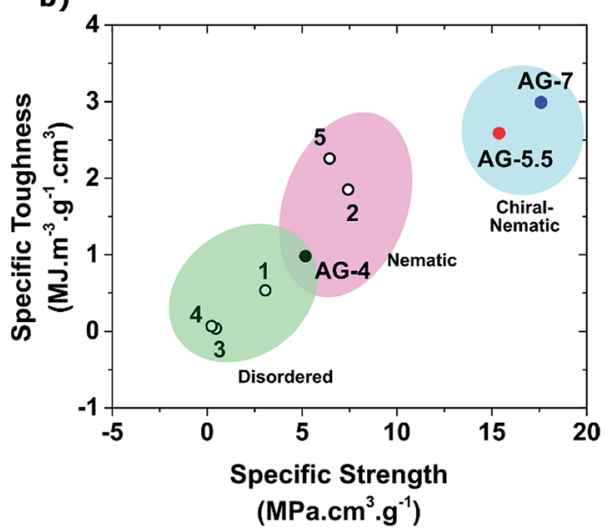

c)

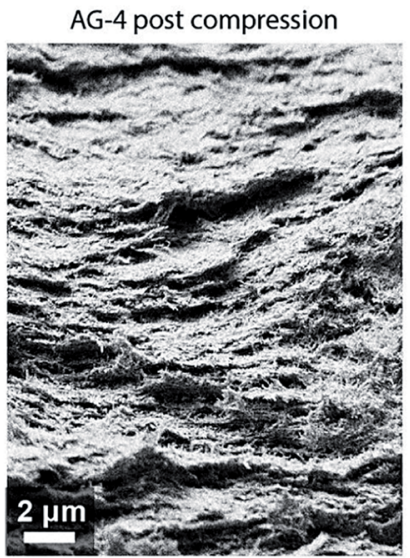

AG-7 post compression

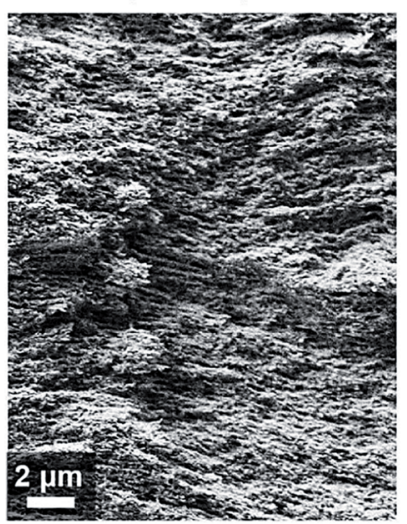

EISA-25 post compression

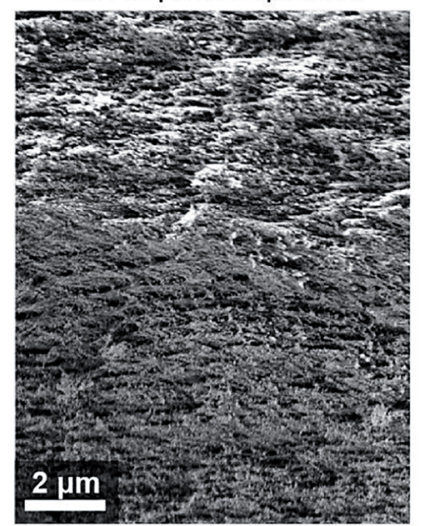

Fig. 4 (a) Stress-strain curves of the CNC aerogels. The inset shows the magnification of stress-strain curves at low strains; compression dependent development of structural color for $\mathrm{cn}$ domains containing AG-5.5 is shown on the right-hand side for initial, $50 \%$ and maximum compression. Note that the image presented results from an identical light source orientation and intensity to maximize specific reflections. The off-white appearance of the initial aerogel is representative of a white aerogel. (b) A plot of specific toughness vs. specific strength exhibiting the mechanical properties obtained for CNC aerogels in this study (closed circles) compared to those previously reported in the literature (open circles). Note that the values for EISA-10 and EISA-20 also lie within the $c n$ domain of the specific toughness vs. specific strength plot (Fig. S8 $†$ ). (1) Abraham et al., ${ }^{40}$ (2) Plappert et al., ${ }^{21}$ (3) Yang \& Cranston, ${ }^{41}$ (4) Yang et al., ${ }^{42}$ and (5) Kobayashi et al. ${ }^{27}$ (c) Post compression SEM images of the AG4, AG-7, and EISA-25 samples.

properties due to an approximately doubled density when compared to the EISA-10 aerogel. The latter observation highlights a new milestone in the limits of mechanical toughness and strength for nanocellulose constructs.

Insights into the compression-response of the aerogels were further provided by SEM observations of the compressed aerogels. The lower section of the aerogels after compression (Fig. 4c) indicates a completely collapsed structure with a laminar morphology but clearly not a $c n$ order for AG-4. In contrast, AG-7 and EISA-25 underwent a significant reduction in pitch length with a well conserved $c n$ order.

\section{Effect of aerogel architectures and compression on their porous structures and associated CNC arrangements}

The observed compression-induced iridescence and the outstanding performance of $c n$-CNC aerogels further motivated analysis of the pore size distribution as it was previously wellcorrelated with mechanical performance for nematically ordered nanocellulose aerogels. ${ }^{21}$ In this endeavor, the data obtained from $\mathrm{N}_{2}$ adsorption isotherms and $\mathrm{BJH}$ desorption curves (Fig. 5) provide insights into the porous structures and associated CNC arrangements in the aerogels. For all aerogels, $\mathrm{N}_{2}$ adsorption isotherms followed an IUPAC Type IV isotherm attributed to a predominantly mesoporous structure (Fig. 5a for the AG-7 aerogel). The fact that the shape of the isotherm remained unchanged upon aerogel compression indicates that the obtained structures preserve the structure of pores in the size range of 2-100 $\mathrm{nm}$. However, the extent of $\mathrm{N}_{2}$ gas adsorbed by the AG-7 aerogel was maximum at an intermediate level of aerogel compression. This suggests an increase in the pore volume in the $10-100 \mathrm{~nm}$ range, which can be confirmed by a complete analysis as evaluated in Table 1 . In addition, the fact that the BET surface area does not change significantly upon compression suggests that, principally, few pores greater than $100 \mathrm{~nm}$ shrink to pore sizes of less than $100 \mathrm{~nm}$. This is associated with an increase in capillary condensation of $\mathrm{N}_{2}$ as previously demonstrated. ${ }^{21}$

The PSD data and mean pore size values can be seen in Table 1. A lower value of Full Width at Half Maximum (FWHM) 
a)

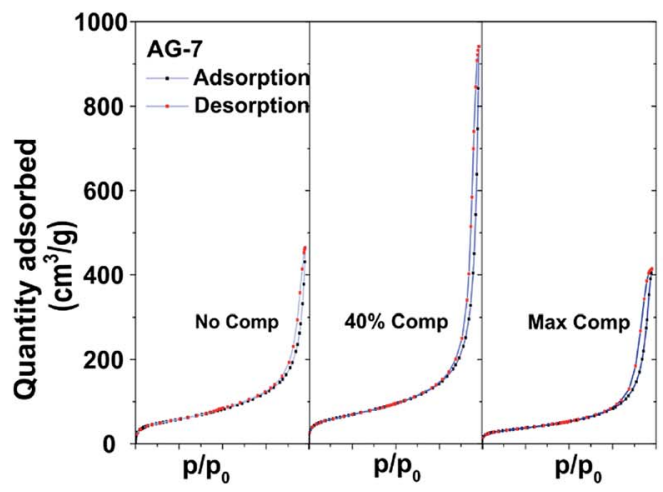

c)

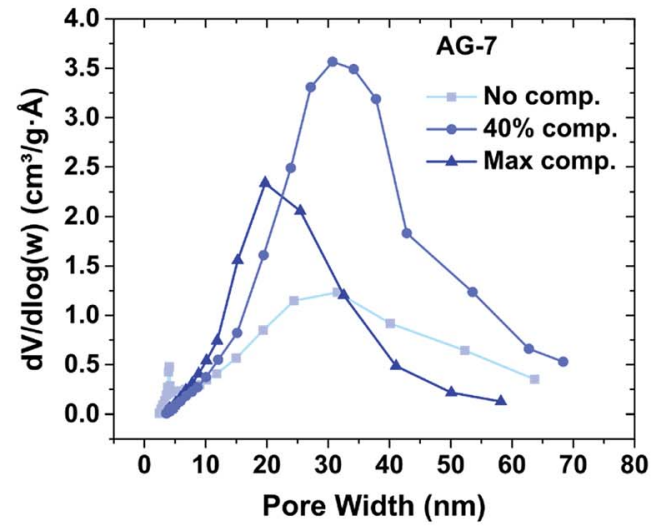

b)

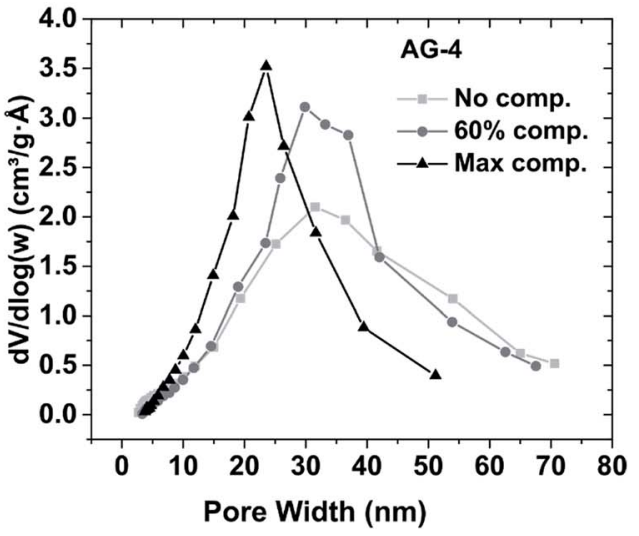

d)

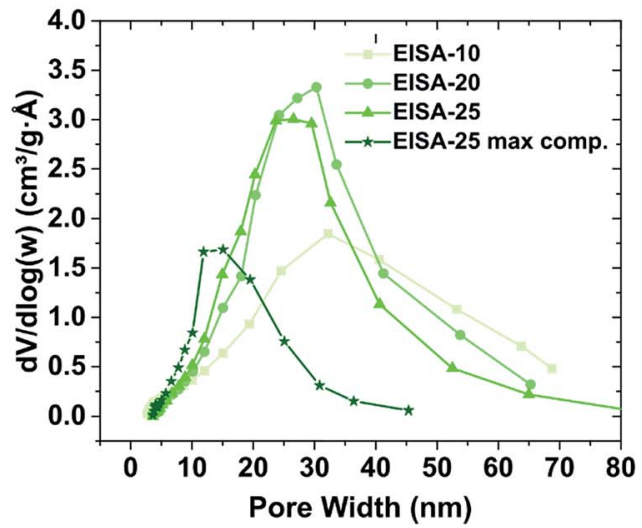

Fig. 5 (a) The $\mathrm{N}_{2}$ adsorption isotherm of the AG-7 aerogel as a function of the strain-dependent compression detailed in Table 1. The pore size distribution (PSD) of AG-4 (b) and AG-7 (c) aerogels as a function of the strain-dependent compression detailed in Table 1. (d) The PSD of EISA-10, 20 and 25 aerogels as well as the effect of the strain-dependent compression detailed in Table 1 on EISA-25.

indicates that pore sizes in the aerogels have a narrower distribution. The broad PSD of AG-4 (38.3 nm, FWHM = 45.9 $\mathrm{nm})$ becomes significantly narrower after intermediate $(32.5 \mathrm{~nm}, \mathrm{FWHM}=22.4 \mathrm{~nm})$ and full compression $(24.3 \mathrm{~nm}$,
FWHM $=17.1 \mathrm{~nm})$, evident from Fig. $5 \mathrm{~b}$. This is in good agreement with the behavior of low-density $\left(18-25 \mathrm{mg} \mathrm{cm}^{-3}\right)$ CNF aerogels. ${ }^{21} \mathrm{~A}$ similar effect was seen for AG-7, where the PSD of the AG-7 aerogel $(33.6 \mathrm{~nm}$, FWHM $=36.1 \mathrm{~nm}$ ) is

Table 1 Properties of CNC aerogels as determined from the $\mathrm{N}_{2}$ adsorption isotherm

\begin{tabular}{|c|c|c|c|c|c|c|c|c|}
\hline \multirow[b]{2}{*}{ Samples } & \multirow{2}{*}{$\begin{array}{l}\text { Comp. } \\
(\%)\end{array}$} & \multirow{2}{*}{$\begin{array}{l}\text { Bulk density } \\
\left(\mathrm{g} \mathrm{cm}^{-3}\right)\end{array}$} & \multirow{2}{*}{$\begin{array}{l}\text { BET area } \\
\left(\mathrm{m}^{2} \mathrm{~g}^{-1}\right)\end{array}$} & \multirow{2}{*}{$\begin{array}{l}\text { Porosity }^{a} \\
(\%)\end{array}$} & \multirow{2}{*}{$\begin{array}{l}\text { Pore volume }{ }^{b} \\
\left(\mathrm{~cm}^{3} \mathrm{~g}^{-1}\right)(2-100 \mathrm{~nm})\end{array}$} & \multicolumn{2}{|c|}{$\begin{array}{l}\text { Pore size } \\
\text { distribution (nm) }\end{array}$} & \multirow{2}{*}{$\begin{array}{l}\mathrm{N}_{2} \text { adsorbed }^{c} \\
\left(\mathrm{~cm}^{3} \mathrm{~g}^{-1} \mathrm{STP}\right)\end{array}$} \\
\hline & & & & & & Mean & FWHM & \\
\hline \multirow[t]{3}{*}{ AG-4 } & 0 & 0.137 & 255 & 91.4 & 7.9 & 38.3 & 45.9 & 735 \\
\hline & 60 & 0.343 & 213 & 78.6 & 9.3 & 32.5 & 22.4 & 791 \\
\hline & Full (89\%) & 1.245 & 232 & 22.2 & 7.5 & 24.3 & 17.1 & 797 \\
\hline & Full (72.6\%) & 0.726 & 196 & 54.6 & 6.4 & 23.1 & 17.8 & 412 \\
\hline EISA-10 & 0 & 0.171 & 231 & 89.3 & 6.4 & 38.6 & 46.5 & 472 \\
\hline EISA-20 & 0 & 0.329 & 249 & 79.4 & 9.3 & 28.8 & 20.8 & 889 \\
\hline EISA-25 & 0 & 0.428 & 240 & 73.2 & 10.9 & 26.6 & 20.7 & 843 \\
\hline EISA-25 & Full (52.4\%) & 0.899 & 189 & 43.8 & 5.2 & 16.5 & 12.9 & 480 \\
\hline
\end{tabular}

${ }^{a}$ Calculated from bulk density of the aerogel. ${ }^{b}$ From BJH desorption. ${ }^{c}$ From the $\mathrm{N}_{2}$ desorption isotherm at $p / p_{0}=0.972 ; \mathrm{FWHM}=$ full width at half maximum. 
narrowed after intermediate $(32.6 \mathrm{~nm}, \mathrm{FWHM}=23.5 \mathrm{~nm})$ and full compression (23.1 nm, FWHM = $17.8 \mathrm{~nm}$ ), Fig. 5c. It is noteworthy that upon intermediate compression, both AG-4 and AG-7 achieve similar density, porosity, and PSD, yet the pore volume of AG-7 is higher than that of the AG-4 aerogel.

The BJH desorption curves for aerogels obtained after EISA (Fig. 5d) provide another point of comparison for the effect of anisotropicity in the dispersions used as precursors for the aerogels. Compared to that for the aerogels formed from lower concentration dispersions, a narrower PSD is evident for EISA20 and 25, with a mean PSD of 26-29 nm and FWHM of $\sim 20.7 \mathrm{~nm}$, potentially from tightly stacked nematic planes with a reduced pitch of the $c n$ assembly. Upon full compression, the PSD of EISA-25 was reduced to a mean value of $16.5 \mathrm{~nm}$ with a narrower FWHM of $12.9 \mathrm{~nm}$.

\section{Pore volume and structure}

The pore volume obtained from BJH desorption provides additional insights into the development of pores with a radius in the range of 2-100 $\mathrm{nm}$ upon compression. The pore volume achieves a maximum at intermediate compression for AG-4 (9.3 $\left.\mathrm{cm}^{3} \mathrm{~g}^{-1}\right)$ and AG-7 (11.1 $\mathrm{cm}^{3} \mathrm{~g}^{-1}$ ), which again suggests pore size harmonization at the expense of macropores to pores within 2-100 nm, as shown in Fig. 6. Upon maximum compression, a decrease in pore volume is observed. However, the decrease in pore volume is more prominent for the AG-7 aerogel. A similar behavior in pore structure transition was observed by Plappert et al. ${ }^{21}$ after densification of nematic aerogels formed from periodate oxidized CNFs.

In the case presented herein, the AG- 4 aerogel (78\% isotropic volume fraction), forms randomly aggregated CNC structures. They are revealed to be disordered, quasi-cellular pore structures represented as crisscross arrangements in Fig. 6. The AG-7 aerogel formed from a nearly fully anisotropic $7 \%$ CNC dispersion exhibits an ordered pore structure throughout the aerogel volume (represented as stacked lines in Fig. 6). The large distance between CNCs in AG-4 may result in clustering prior to gelation. Upon intermediate compression and owing to the more continuous and ordered network of pores in AG-7, collapse of pores in AG-7 occurs less easily than in AG-4. This also limits the $\mathrm{N}_{2}$ accessibility and its condensation in the pores of the AG-4 aerogel. The pore-collapse behavior also explains why a dramatic increase in pore volume for AG-7 is observed compared to that for AG-4, although the mean value of PSD is approximately similar for both.

The latter observations are in contrast with those for EISA-10 to EISA-25, which results in a densification by EISA rather than by compression. Hence, the pore volume increases from 6.4 to $10.9 \mathrm{~cm}^{3} \mathrm{~g}^{-1}$ with a small reduction in porosity from 89.3 to $73.2 \%$. The results suggest that the dispersion gelled and developed a more close-packed long-range order by evaporation, which translated into an ordered and compact architecture with uniform and small pore sizes for the obtained aerogels. Upon full compression of EISA-25, both the porosity and pore volume were reduced to 10.9 and $5.2 \mathrm{~cm}^{3} \mathrm{~g}^{-1}$, respectively, suggesting that some pores within the size range of 2-100 $\mathrm{nm}$ were either fully collapsed or became inaccessible. Additionally, the $\mathrm{N}_{2}$ adsorption of films produced with the same CNC mass via full EISA did not reveal any mesoporous structures (Fig. S9†). This information coupled with the compression data of AG-7 and EISA-25 suggests a limit in self-assembly/ compression effects where mesopores cannot be maintained above $25 \%$ for well-ordered CNC aerogels.

Aerogels obtained from precursors at high CNC concentrations by EISA further self-assemble and may be more beneficial for generating uniform mesoporous structures and tough aerogels without additional compression. This is in contrast, to some extent, to the behavior observed for AG-7, where an intermediate compression is highly beneficial for the

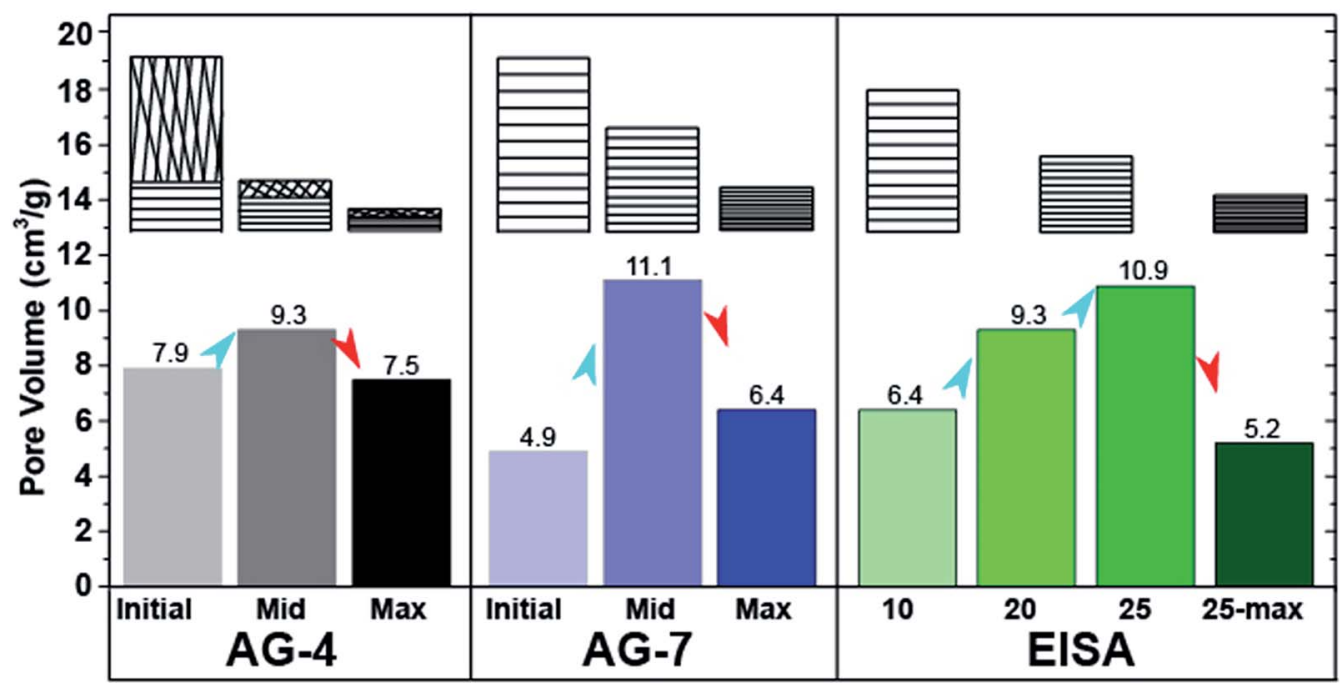

Mesopore increase

>Pore collapse

Fig. 6 The pore volume in the pore range of 2-100 nm, calculated from the BJH desorption isotherm. The illustrations demonstrate the effect of compression on the isotropic (crisscrossed lines) and anisotropic (linear lines) fractions of the aerogels. 
generation of uniform pores with sizes of 2-100 $\mathrm{nm}$. Overall, a tightly packed $c n$ structure resulting from an incomplete EISA or compression of a 'looser' aerogel with $c n$ order (e.g. AG-7) generated uniform mesoporous structures and may present enhanced strength and toughness, as seen from EISA-10 and 25 and intermediate compression of AG-7.

\section{Conclusions}

A range of light-weight aerogels that preserve the $c n$ structure of the precursor CNC dispersions as well as their order were produced. We demonstrate a strong correlation between increasing the $c n$ arrangement of CNCs and enhancing the mechanical robustness of the aerogels. In fact, the specific strength and toughness of the micro-structurally ordered CNC aerogels, two generally mutually exclusive properties, are $\sim 500-$ $600 \%$ higher than those of disordered CNC aerogels reported in the literature. Aerogels from predominantly isotropic $4 \% \mathrm{CNC}$ dispersions (AG-4) exhibit more disordered structures, and hence lower specific strength and toughness ( $\sim 3$ times), compared to aerogels formed from completely anisotropic $7 \%$ CNC aqueous dispersions (AG-7). Upon enhancing the ordering of CNCs via evaporation induced self-assembly (EISA), a further increase in mechanical properties is observed with specific strength and toughness reaching 21.5 and $3.5 \mathrm{MPa} \mathrm{cm}^{3} \mathrm{~g}^{-1}$, respectively for the EISA-10 aerogel. The absolute value of strength and toughness increases for EISA-20 but a two-fold increase in density decreases the specific values of strength and toughness. The aerogel pore structure revealed that uniaxial densification more than doubles the pore volume (2$100 \mathrm{~nm}$ ) of the AG-7 aerogels by partially compressing the nematically arranged macropores into a range of smaller pores within the $2-100 \mathrm{~nm}$ range. Such a dramatic increase in pore volume was not observed for compression of disordered CNC arrangements in AG-4 aerogels. In contrast, the EISA-10 to EISA25 aerogels exhibited a gradual increase in pore volume as the dispersion was concentrated by EISA. Finally, the compression of $c n$-CNC aerogels exhibited structural color arising from the $c n$ orientation of CNCs, which is strongly coupled with the mechanical robustness of $c n$-CNC aerogels, thus making them attractive choices as colorimetric sensors, for instance, for mechanically damaged goods.

The increased anisotropy, long-range order in the aerogels, is strongly correlated with an enhanced specific strength and specific toughness. Therefore, our results encourage the use of highly ordered porous structures via long-range ordering of nano-scale building blocks to form ultra-robust materials, effectively expanding the property space of CNC aerogels. Besides the properties of the formed aerogels, including mechanically responsive iridescence and high pore volume, this study opens new areas of application for porous CNC architectures and also new considerations for the development of porous constructs from liquid crystals.

\section{Conflicts of interest}

There are no conflicts to declare.

\section{Acknowledgements}

A. T. and B. L. T. contributed equally to the study. We acknowledge funding from the European Research Council (ERC) under the European Union's Horizon 2020 Research and Innovation Program (grant agreement No 788489).

\section{Notes and references}

1 U. G. Wegst, H. Bai, E. Saiz, A. P. Tomsia and R. O. Ritchie, Nat. Mater., 2014, 14, 23-26.

2 H. Sehaqui, M. Salajková, Q. Zhou and L. A. Berglund, Soft Matter, 2010, 6, 1824.

3 A. Tripathi, G. N. Parsons, S. A. Khan and O. J. Rojas, Sci. Rep., 2018, 8, 1-12.

4 A. Tripathi, S. A. Khan, O. J. Rojas, G. N. Parsons, J. K. Steach, J. S. De Witt, S. M. B. Islam, J. D. Goodrich, WO 2017127828 A1, 2017.

5 N. T. Cervin, L. Andersson, J. B. S. Ng, P. Olin, L. Bergström and L. Waišgberg, Biomacromolecules, 2013, 14, 503-511.

6 M. Aegerter, N. Leventis and M. Koebel, Aerogels handbook (Advances in Sol-Gel Derived Materials and Technologies), 2011.

7 B. M. Novak, D. Auerbach and C. Verrier, Chem. Mater., 1994, 6, 282-286.

8 J. Song, C. Chen, S. Zhu, M. Zhu, J. Dai, U. Ray, Y. Li, Y. Kuang, Y. Li, N. Quispe, Y. Yao, A. Gong, U. H. Leiste, H. A. Bruck, J. Y. Zhu, A. Vellore, H. Li, M. L. Minus, Z. Jia, A. Martini, T. Li and L. Hu, Nature, 2018, 554, 224-228.

9 J. Song, C. Chen, Z. Yang, Y. Kuang, T. Li, Y. Li, H. Huang, I. Kierzewski, B. Liu, S. He, T. Gao, S. U. Yuruker, A. Gong, B. Yang and L. Hu, ACS Nano, 2018, 12, 140-147.

10 T. Li, J. Song, X. Zhao, Z. Yang, G. Pastel, S. Xu, C. Jia, J. Dai, C. Dai, A. Gong, F. Jiang, Y. Yao, T. Fan, B. Yang, L. Wågberg, R. Yang and L. Hu, Sci. Adv., 2018, 4, 1-10.

11 C. A. de Assis, M. C. Iglesias, M. Bilodeau, D. Johnson, R. Phillips, M. S. Peresin, E. M. T. Bilek, O. J. Rojas, R. Venditti and R. Gonzalez, Biofuels, Bioprod. Biorefin., 2018, 12, 251-264.

12 C. A. de Assis, C. Houtman, R. Phillips, E. M. T. Bilek, O. J. Rojas, L. Pal, M. S. Peresin, H. Jameel and R. Gonzalez, Biofuels, Bioprod. Biorefin., 2017, 11, 682-700.

13 N. Lavoine and L. Bergström, J. Mater. Chem. A, 2017, 5, 16105-16117.

14 J. F. Lutz, J. M. Lehn, E. W. Meijer and K. Matyjaszewski, Nat. Rev. Mater., 2016, 1, 16024.

15 K. J. De France, T. Hoare and E. D. Cranston, Chem. Mater., 2017, 29, 4609-4631.

16 C. Salas, T. Nypelö, C. Rodriguez-Abreu, C. Carrillo and O. J. Rojas, Curr. Opin. Colloid Interface Sci., 2014, 19, 383396.

17 Y. Zhang, T. Nypelö, C. Salas, J. Arboleda, I. C. Hoeger and O. J. Rojas, J. Renewable Mater., 2013, 1, 195-211.

18 D. Klemm, F. Kramer, S. Moritz, T. Lindström, M. Ankerfors, D. Gray and A. Dorris, Angew. Chem., Int. Ed., 2011, 54385466. 
19 Y. Habibi, L. A. Lucia and O. J. Rojas, Chem. Rev., 2010, 110, 3479-3500.

20 A. Dufresne, Mater. Today, 2013, 16, 220-227.

21 S. F. Plappert, J. M. Nedelec, H. Rennhofer, H. C. Lichtenegger and F. W. Liebner, Chem. Mater., 2017, 29, 6630-6641.

22 T. Liebert, Cellulose Solvent: For Analysis, Shaping and Chemical Modification: 1 Cellulose Solvents- Remarkable History, Bright Future, ACS Symposium Series, 2010.

23 B. D. Mattos, L. G. Greca, B. L. Tardy, W. L. E. Magalhães and O. J. Rojas, Small, 2018, 14, 1-10.

24 F. J. Parmenter and K. E. Milstein, J. Non-Cryst. Solids, 1998, 223, 179-189.

25 J. P. F. Lagerwall, C. Schütz, M. Salajkova, J. Noh, J. Hyun Park, G. Scalia and L. Bergström, NPG Asia Mater., 2014, 6, e80.

26 K. W. Klockars, B. L. Tardy, M. Borghei, A. Tripathi, L. G. Greca and O. J. Rojas, Biomacromolecules, 2018, 19, 2931-2943.

27 Y. Kobayashi, T. Saito and A. Isogai, Angew. Chem., Int. Ed., 2014, 53, 10394-10397.

28 B. L. Tardy, M. Ago, J. Guo, M. Borghei, T. Kämäräinen and O. J. Rojas, Small, 2017, 13, 1702084.

29 M. Gu, C. Jiang, D. Liu, N. Prempeh and I. I. Smalyukh, ACS Appl. Mater. Interfaces, 2016, 8, 32565-32573.

30 B. Frka-Petesic, G. Guidetti, G. Kamita and S. Vignolini, Adv. Mater., 2017, 29, 1701469.
31 B. L. Tardy, B. D. Mattos, L. G. Greca, T. Kämäräinen, K. W. Klockars and O. J. Rojas, Adv. Funct. Mater., 2019, 1808518.

32 B. Natarajan and J. W. Gilman, Philos. Trans. R. Soc., A, 2018, 376, 20170050.

33 J. C. Weaver, G. W. Milliron, A. Miserez, K. Evans-Lutterodt, S. Herrera, I. Gallana, W. J. Mershon, B. Swanson, P. Zavattieri, E. DiMasi and D. Kisailus, Science, 2012, 336, 1275-1280.

34 N. Suksangpanya, N. A. Yaraghi, D. Kisailus and P. Zavattieri, J. Mech. Behav. Biomed. Mater., 2017, 76, 38-57.

35 M. Mitov, Soft Matter, 2017, 13, 4176-4209.

36 Y. T. Xu, Y. Dai, T. D. Nguyen, W. Y. Hamad and M. J. MacLachlan, Nanoscale, 2018, 10, 3805-3812.

37 A. Hirai, O. Inui, F. Horii and M. Tsuji, Langmuir, 2009, 25, 497-502.

38 J. Noh, J. P. F. Lagerwall, G. Scalia, C. Schütz, J. H. Park, G. Salazar-Alvarez and L. Bergström, ChemPhysChem, 2014, 15, 1477-1484.

39 M. Giese, M. K. Khan, W. Y. Hamad and M. J. MacLachlan, ACS Macro Lett., 2013, 2, 818-821.

40 E. Abraham, D. E. Weber, S. Sharon, S. Lapidot and O. Shoseyov, ACS Appl. Mater. Interfaces, 2017, 9, 2010-2015.

41 X. Yang and E. D. Cranston, Chem. Mater., 2014, 35, 60166025.

42 X. Yang, K. Shi, I. Zhitomirsky and E. D. Cranston, Adv. Mater., 2015, 27, 6104-6109. 